\title{
Téoros
}

Revue de recherche en tourisme

\section{Le tourisme culturel en Turquie}

La question du même et la question de l'Autre

\section{Nilgun Tutal}

Volume 22, numéro 3, automne 2003

URI : https://id.erudit.org/iderudit/1071347ar

DOI : https://doi.org/10.7202/1071347ar

Aller au sommaire du numéro

Éditeur(s)

Université du Québec à Montréal

ISSN

0712-8657 (imprimé)

1923-2705 (numérique)

Découvrir la revue

\section{Citer cet article}

Tutal, N. (2003). Le tourisme culturel en Turquie : la question du même et la question de l'Autre. Téoros, 22(3), 42-49. https://doi.org/10.7202/1071347ar d'utilisation que vous pouvez consulter en ligne.

https://apropos.erudit.org/fr/usagers/politique-dutilisation/ 


\section{Le tourisme culturel en Turquie La question du même et la question de l'Autre}

\section{Nilgun Tutal}

A vec ses plages ensoleillées, ses formules d'hébergement à bon marché, son riche patrimoine historique et les légendes qui l'entourent, l' « Orient » demeure, en dépit des crises, un marché touristique des plus attractifs. En 2001, les pays du Maghreb, du Proche-Orient et d'Asie mineure accueillaient ainsi près de trente millions de visiteurs, pour la plupart originaires d'Europe et d'Amérique du Nord (OMT, 2001). Pour le touriste, le choix d'une destination « orientale » n'est pas anodin : les paramètres financiers entrent bien sûr en ligne de compte, mais l'image que le vacancier se fait de la région ou du pays qu'il souhaite visiter joue ici un rôle capital. L'image de 1 '« Orient » se construit en partie sur des textes et des images de promotion touristique : publicités, guides touristiques, articles de presse... La présente étude porte sur les images de l' «Orient » véhiculées par cette dernière catégorie.

Les articles touristiques de la presse généraliste ont en effet pour particularité de s'intéresser plus que les textes publicitaires aux aspects « culturels » des pays visités. Le souci d'objectivité journalistique les conduit en outre à introduire dans leur discours des éléments dépréciatifs de l'objet décrit et à prendre en compte le contexte politique du pays en question, alors que les publicités et les guides touristiques évitent, autant que faire se peut, d'évoquer ces aspects ; autant de facteurs qui permettent de croire que l'analyse des articles de presse permet d'accéder à des images plus variées et plus élaborées de l' «Orient» que celles des textes publicitaires et nous les considérons comme autant de représentations collectives.

Il faut ici entendre l' «Orient » dans le sens que lui assigne Saïd (1997 : 13), en lien avec l'orientalisme «qui est une manière de s'arranger avec l'Orient fondée sur la place particulière que celui-ci tient dans l'expérience de l'Europe occidentale ». Pour cet auteur, cet espace à la géographie incertaine «n'est pas seulement le voisin immédiat de l'Europe, c'est aussi la région où l'Europe a créé les plus vastes, les plus riches et les plus anciennes de ses colonies, la source de ses civilisations et de ses langues; il lui fournit l'une des images de l'Autre qui s'impriment le plus profondément en lui. De plus, l'Orient a permis de définir l'Europe (ou l'Occident) par contraste : son idée, son image, sa personnalité, son expérience » (1997 : 13-14). À l'instar de Saïd, nous utilisons la notion de représentation en référence aux représentations de l'Autre imaginairement construites par une culture donnée. Nous pensons que c'est grâce à cet imaginaire qu'une culture signifie le monde qui l'entoure. Selon la sociologue Ruth Amossy d'ailleurs, c'est par rapport à cet imaginaire qu' «un va-et-vient s'établit ainsi entre les images logées 'dans notre tête' et celles que divulguent abondamment les textes et les médias » (1991: 10) sur les cultures étrangères. Ainsi, la prise de parole sur l'Autre par les membres d'une culture mobilise certaines représentations et fait entrer en jeu son propre imaginaire collectif. Cet imaginaire est composé de représentations culturelles qui, selon Uli Windisch, sociologue, sont communiquées de façon répétée et peuvent même finir par être distribuées dans le groupe entier, c'est-à-dire faire l'objet d'une version mentale dans chacun de ses membres. Les représentations qui sont ainsi largement distribuées dans un groupe social et l'habitent de façon durable sont des représentations culturelles (1989 : 177).

En effet, les mythes, les légendes populaires, les conceptions religieuses de toute sorte, les conceptions morales, etc., construisent la mémoire imaginaire, qui fait appel à des représentations qu' Admiral et Lipiansky, linguistes, définissent comme celles «qui s'ancrent dans un imaginaire social, fruit de l'histoire et des rapports entre groupes ethniques ou nationaux » (1989:199).

Nous ne sommes pas en mesure, dans le cadre de notre étude, d'aborder l'ensemble des représentations de l' «Orient » dans les médias « occidentaux ». Aussi avonsnous choisi de restreindre notre champ d'analyse aux articles publiés par la presse française $^{2}$ pendant la période allant de 1987 à 1999 et se rapportant à un seul pays, la Turquie. Le choix de ce pays se justifie par ses relations privilégiées avec l'Europe. Située à la charnière entre les continents européen et asiatique, engagée depuis le $\mathrm{XIX}^{\mathrm{e}}$ siècle dans un processus sans équivalent d'occidentalisation qui l'a conduite à prendre l'Europe pour modèle dans les domaines politique, économique et culturel, la République turque est en effet officiellement candidate depuis 1963 à l'adhésion à l'Union européenne (UE, à cette époque la Communauté économique 
européenne). Notre intérêt réside dans le fait que, depuis lors, politiciens et médias occidentaux n'ont eu de cesse de scruter l'identité de la nation turque pour en déterminer les caractères orientaux ou européens. La question récurrente de la candidature turque a également conduit les dirigeants européens à s'interroger sur l'identité de l'Union européenne et sur ce qui n'est pas l'Europe, c'est-à-dire son plus proche voisin, l'« Orient ». C'est d'ailleurs la prééminence de la France dans ces débats - on se souviendra notamment des déclarations de Valery Giscard d'Estaing, président de la Convention européenne, sur la dimension chrétienne de l'Europe - et son rôle dans la définition de la politique étrangère de l'UE qui nous ont amenés à choisir la presse française comme objet d'étude.

Dans un tel contexte, le choix de la période analysée n'est pas anodin. L'année 1987 correspond au renouvellement de la candidature turque et à la reprise des relations avec la Communauté économique européenne après l'interruption en Turquie du processus démocratique consécutive au coup d'État militaire de 1980. Par ailleurs, 1999 est l'année du Sommet de Helsinki, au cours duquel les dirigeants des Quinze ont confirmé la vocation de la République turque à intégrer l’Union européenne.

Notre choix de la Turquie comme objet du discours médiatique analysé répond par ailleurs à des considérations d'ordre touristique. Parmi les pays que nous avons décrits comme relevant de l' «Orient», la République turque accueille en effet à elle seule près du tiers du flot touristique avec 10 millions de visiteurs en 2001, loin devant l'Égypte (5 millions), la Tunisie (5 millions) ou le Maroc (4 millions). Ses revenus touristiques (OMT, 2001) dépassent ainsi 10 milliards de dollars annuellement ${ }^{3}$. Les chiffres fournis par le ministère turc du tourisme pour les six premiers mois de 2002 confirment en outre la provenance majoritairement européenne de ces touristes : sur 1462795 visiteurs, 970464 viennent des pays de l'Union européenne, principalement d'Allemagne, d'Autriche, de Belgique, du Danemark, de Finlande et de
France $^{4}$. Les touristes européens visitent en particulier trois régions turques : la mer de Marmara et la ville d'Istanbul ; la mer Egée ; les côtes de la mer Méditerranée. À elles seules, ces trois régions accueillent 91,3\% des touristes européens venus en Turquie $^{5}$. Cet attrait des vacanciers européens pour la Turquie se traduit dans les médias par un nombre important d'articles touristiques qui vantent la richesse du patrimoine historique turc.

\section{À la recherche de Soi}

La curiosité à l'égard de l'Autre se manifeste aujourd'hui à travers le tourisme de masse comme la recherche d' " une consommation sans cesse renouvelée de l'exotique' » (Kilani, 1996 : 7). Ce phénomène est, comme le définit Lanfant (1994 : 434), une pratique « qui fait sentir ses effets de proche en proche dans tous les secteurs de la vie collective: dans les institutions, les mentalités, les conceptions de l'identité, et jusqu'à l'idée que la société se fait d'elle-même ». Ce processus fait entrer la culture, le patrimoine historique, les traditions, l'identité même dans la catégorie des marchandises. Le patrimoine historique d'un pays devient de ce fait une matière première à exploiter. La rhétorique constitutive de ce processus insiste sur le fait qu'il existe des identités à retrouver et à célébrer. L'activité touristique se présente ainsi comme «le support d'un retour aux sources, d'un itinéraire vers les racines et les lieux de mémoire » (Lanfant, 1994 : 437).

Ce processus de marchandisation du patrimoine historique et culturel, généré par le phénomène touristique, prend en Turquie un sens particulier : l'appropriation du territoire turc et l'exclusion du peuple qui l'habite sont en effet les deux facettes de la découverte de la Turquie dans le discours touristique français.

\section{La recherche et l'appropriation du patrimoine historique anatolien}

De nos jours, découvrir la Turquie revient à découvrir la richesse historique et culturelle de l'Europe.
Les origines de la Civilisation universelle On peut lire dans Le Figaro Magazine du 4 juin 1994 les raisons pour lesquelles la Turquie vaut d'être visitée : «Depuis des siècles, la terre turque a fait éclore une infinité de richesses. Pour le voyageur, c'est aujourd'hui un lieu magique où se ressourcer. Découvrez en Turquie cette richesse culturelle et historique de l'Europe ». La Turquie est ainsi considérée comme un espace historique où le visiteur européen peut retrouver ses origines culturelles et reconstruire son passé. Cette quête des origines est formulée comme un besoin par le magazine touristique Géo : «La civilisation occidentale a besoin de se ressourcer et de redécouvrir ses plus lointains ancêtres : la Turquie d'avant les Turcs mérite à elle seule une grande exploration » (Géo, 1987 : 71).

Nous assistons ici à l'appropriation du patrimoine historique du territoire turc par un « nous » qui fait référence à la Civilisation universelle présentée comme un apanage essentiel de l'Europe, mais qui ne relève pas de la culture turque ; c'est « la Turquie d'avant les Turcs » qui intéresse le journaliste. Ce mécanisme est plus explicite encore dans l'énoncé suivant, qui décrit une ville préhistorique du centre de la Turquie : «Les vestiges d'une véritable ville de cinq cents mètres de long, juchée à vingt mètres au-dessus de la plaine ; la plus ancienne cité de l'Occident (-9000) construite selon un plan » (Coutin, 1987 : 90).

Comment une cité d'Asie mineure vieille de 11000 ans peut-elle être qualifiée d' « occidentale »? La conception française de la civilisation, telle qu'elle a été mise en lumière par Admiral et Lipianski, peut nous permettre d'expliquer cet anachronisme :

la France décrit son visage comme celui de la civilisation, elle ne parle pas de civilisation française, mais de civilisation tout court [...] Si le Français s'élève sans effort du particulier à l'universel, c'est qu'en lui déjà le particulier a pris la forme universelle de la raison. La civilisation n'est pas, pour lui, l'apanage d'un temps ou d'un pays : elle est 
l'œuvre commune et le bien propre de l'humanité [...] La France ne cherche pas à imposer sa civilisation, elle combat pour un idéal universel (1989: 104).

Si le Français est capable de « s'élever sans effort du particulier à l'universel », il lui est non moins aisé d'assimiler la Civilisation universelle à la sienne propre, qu'il nomme cette dernière occidentale ou européenne. Le fait que la cité préhistorique décrite ci-dessus ait été construite selon un plan, c'est-à-dire selon un schéma organisé et rationnel, suffit pour l'inscrire dans l'espace de la civilisation universelle/occidentale.

Les différentes désignations du territoire turc employées dans les articles analysés viennent conforter l'idée d'appropriation d'un espace et d'un passé. Les dénominations anciennes de certains sites, évocatrices de leur passé chrétien, sont en effet souvent préférées à leur nom turc actuel : Ararat pour Agri Dagi, Andrinople pour Edirne, Constantinople pour Istanbul, Smyrne pour Izmir, Brousse pour Bursa... Selon Étienne Copeaux, turcologue, cette préférence pour les toponymes anciens « a le mérite d'attirer l'attention sur une certaine vision occidentale de l'Anatolie, intéressée par l'Antiquité classique, revendiquée comme passé de l'Occident» (1997 : 289). Il est d'avis que les Turcs se montrent pour leur part hostiles à l'utilisation de ces toponymes anciens et s'efforcent de faire valoir leur équivalent turcisé. La vision turque de l'Antiquité grecque tend également à relativiser «le mérite historique grec considéré comme grandement exagéré par l'historiographie européenne. Le discours historique concernant la Grèce classique et l'Antiquité anatolienne est conçu comme une réfutation de la formule célèbre 'le miracle grec'»(Copeaux, 1997 : 293).

Qu'elle soit appelée dans le discours européen Anatolie, Asie mineure ou, plus souvent, Mésopotamie, la Turquie est rarement décrite comme la terre des Turcs. Derrière la dénomination de Mésopotamie se cache ainsi « le berceau du monde, l'entre-deux fleuves » où se réalise « un brassage continuel de civilisations ». C'est en effet dans la haute vallée du Tigre et de l'Euphrate que l'Europe moderne trouve un certain nombre de ses références historiques : «Pour les Européens, c'est l'Orient, mais au sens figuré. Le soleil qui s'y est levé se trouve à l'origine de notre univers spirituel, de nos croyances religieuses, de notre civilisation » (Schneider, 1987 : 78). En reconnaissant l'antériorité des civilisations mésopotamiennes et en se réclamant de leur postérité, l'Europe affirme par ailleurs sa propre profondeur historique.

Ce droit à revendiquer l'héritage des civilisations passées se trouve dans le même temps refusé aux Turcs quand ceux-ci veulent en user pour attester de leur vocation à devenir Européens. Aussi, peut-on lire dans un article de François Bécet, publié en 1995 dans le journal régional l'Alsace :

Comment ne pas prendre au sérieux la candidature turque? Les Turcs savent leur importance et en jouent. Le ministère qui s'occupe des investissements le dit, carte à l'appui, dans une publicité : "la Turquie n'est pas le centre du monde [...] elle y est située, tout simplement ». Sans aucun doute, le pays de Mustafa Kemal est incontournable. Mais il n'y pas que la géographie et la géopolitique.

L'auteur de ce texte accepte ainsi que le territoire turc soit situé au « centre du monde », une conception qui évoque la vision classique de l'Anatolie comme le lieu géographique où se rejoignent les grandes civilisations antiques. Il n'en sous-entend pas moins que les Turcs restent, pour une raison ou une autre, différents des Européens et sont non fondés quand ils tentent de se prévaloir du passé du territoire qu'ils habitent.

On le voit ici, le voyage en Turquie tel qu'il est proposé par les articles touristiques de la presse française ressemble plus à une pérégrination intérieure qu'à la rencontre et au mélange avec d'autres cultures. La visite des nombreux vestiges gréco-romains et chrétiens présents sur le territoire turc constitue l'étape suivante sur le chemin de l'intégration des civilisations passées dans l'imaginaire collectif français.

\section{Les vestiges de la civilisation gréco-romaine et du christianisme}

En Turquie, le touriste français, suivant les conseils de ses compatriotes journalistes, partira tout d'abord à la recherche des mythes fondateurs de sa culture avec la Bible et l'Iliade pour guides touristiques :

C'est l'arche de Noé que l'on cherche en vain sur les pentes du Mont Ararat haut de 5.165 mètres. C'est Ilion, plus connu sous le nom de Troie, qui résista dix ans aux assauts d'Agamemnon [...] Voici Izmir la Belle (Smyrne) où, dit-on, Homère est né et a habité (Lacontre, 1995 : 88).

La quête de Soi commence ainsi par la visite de lieux mythiques, que l'imaginaire français et les articles de presse rangent tantôt dans le domaine de la fiction, tantôt dans celui de la réalité. Quand le touriste aborde ensuite un lieu bien réel, comme la ville d'Istanbul, c'est également le présent byzantin et chrétien de cette dernière qu'il vient chercher :

Toute perception d'Istanbul commence par une question d'histoire : Que symbolisait exactement Constantinople pour l'imaginaire occidental? Cette capitale de l'Empire romain d'Orient n'était pas seulement devenue avec Constantin, qui lui donna son nom, celle de la deuxième Rome de la Chrétienté. Elle fut surtout quelque chose comme le lieu géométrique de la civilisation (Guillebaud, 1994 : 85).

Comme on peut l'observer dans ce texte, le discours touristique de la presse française met souvent l'accent sur le passé romain ou byzantin de la ville d'Istanbul. On y trouve en outre l'idée que cette métropole a représenté, dans une époque passée, la civilisation universelle et que c'est cet aspect qui donne valeur à sa visite.

Que l'on reste à Istanbul ou que l'on se déplace vers les villes antiques fondées 


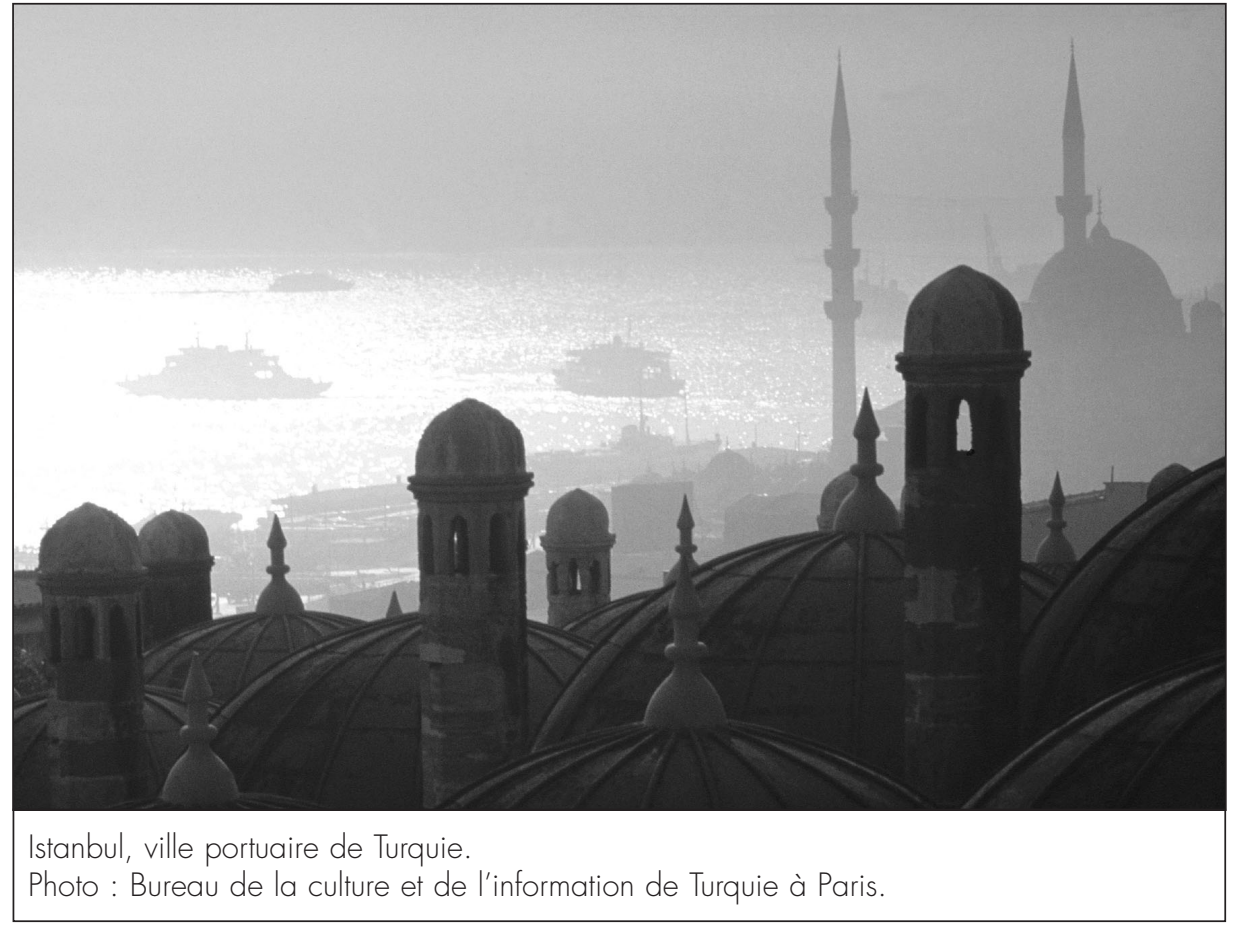

par les Grecs le long des côtes égéennes et méditerranéennes, on voit poindre ce même argument, fortement teinté d'eurocentrisme, qui attribue au génie grec, ancêtre supposé de la culture européenne, la paternité de la civilisation universelle : «Le génie grec y (en Anatolie) forgea superbement les armes de sa puissance civilisatrice [...] Les Grecs en ces mêmes lieux exportèrent leur culture. L'humanisme européen a expérimenté ses modèles sur la côte turque occidentale et méridionale » (Coutin, 1987 : 100). Ce mythe du Grec à l'origine de la civilisation est le fil conducteur du discours touristique. Le reste de la Turquie, c'est-à-dire la portion de territoire qui n'a pas accueilli les cités grecques, est caractérisé avec l'Orient tout entier par les termes de gigantisme, d'esprit de conquête et de despotisme, en opposition à la création civilisatrice et à la démocratie qui relèvent de la Grèce antique, donc de l'Europe.

\section{L'exclusion des Turcs du patrimoine}

Le discours touristique produit par la presse française affiche un intérêt pour toutes les civilisations qui se sont succédé sur le territoire anatolien et pour l'idée d'un brassage des cultures : "Il y a partout des vestiges antiques, témoins des civilisations successives, romaine, chrétienne, byzantine, musulmane, qui ont enrichi ce pays depuis des milliers d'années » (Stoerkel, 1995). Cette pétition de principe est cependant rarement respectée. Les vestiges gréco-romains et chrétiens captent plus le regard des Français que celui de leurs homologues turcs et musulmans.

Istanbul est ainsi présentée comme le lieu par excellence du mélange culturel, de la rencontre entre Orient et Occident : «C'est là, dans la seule ville au monde bâtie sur deux continents, que l'Europe et l'Asie se rencontrent dans le souvenir des empires romain, grec et ottoman » (Prat, 1995). Mais, bien vite, ce sont les liens historiques de cette cité avec l'Europe qui prennent, dans les articles touristiques, le dessus sur ses aspects turcs et musulmans. On se souvient que la ville a été fondée par Constantin, que l'église Sainte-Sophie est le joyau de l'architecture byzantine... L'Istanbul contemporaine, musulmane, s'efface devant les réminiscences de son passé chrétien :

Istanbul s'estompe comme dans le flash-back d'un film. Là s'étalaient jadis, dans la rue de Divan Yolu, les fastes de Byzance la Grecque. Ici viennent d'autres réminiscences, les clameurs des jeux du cirque et des courses de chars sur l'hippodrome de Constantinople, la nouvelle Rome qui survécut mille ans à la chute de son aînée (Héligon, 1994).

La présence de monuments musulmans en vient à gêner l'observateur français dans sa contemplation du passé byzantin de la ville : «En avançant, en direction de la vieille ville, sur le pont de Galata, le regard s'accroche aux cascades de coupoles et aux minarets qui barrent le ciel. Impossible d'ignorer le souvenir de l'époque ottomane » (Prat, 1995). Les Turcs semblent dans certains articles ne pas avoir tout à fait leur place dans ce haut lieu de civilisation :

L'Anatolie demeure encore dans ses profondeurs une vieille terre grecque et byzantine [...] Par milliers [...] ce sont les pierres et les chapiteaux sculptés des édifices bientôt démolis que les Turcs réutiliseront pour bâtir leurs mosquées et leurs propres places fortes [...] Toutes celles que nous visitons portent témoignage de cette mutation $d u$ byzantin au seldjoukide, $d u$ christianisme à l'islam (Guillebaud,1993).

La Turquie est et restera byzantine, semble dire cet extrait, fut-ce à travers les pierres des mosquées construites par ses nouveaux occupants. Alors que les Grecs faisaient figure dans les exemples précédents de bâtisseurs et de propagateurs de la civilisation, les Turcs apparaissent ici comme des destructeurs de ce patrimoine universel. Au bâtisseur s'oppose le destructeur, au civilisé le nomade. Et c'est bien au passé nomade des Turcs que réfèrent plusieurs articles quand ils évoquent le peuple qui habite aujourd'hui la Turquie :

L'Anatolie posait bien des problèmes à un État qui se voulait essentiellement européen [...] Pendant des siècles, les clans résistèrent énergiquement aux mesures prises pour les fixer au sol. Il fallut attendre la seconde moitié du XX $X^{e}$ siècle pour que le nomadisme turc disparut [...] L'Anatolie avait mal admis [...] d'obéir à un prince (Bayezid $1^{\text {er }}$ ) qu'elle 
considérait comme l'héritier des Byzantins (Roux, 1989 : 128).

On trouve la trace du nomadisme turc jusque dans l'architecture des palais ottomans, en l'occurrence celui de Topkapi : "le sérail est un fascinant 'campement' de pierre [...] » (Prat, 1995). Des Seldjoukides qui utilisent les pierres des monuments grecs pour en faire des mosquées aux tribus turques qui refusent de se sédentariser et donc de se civiliser, la perception de l'Autre passe par la mise en évidence de sa différence avec l'Europe. Même s'il ne s'agit pas ici d'un refus catégorique et absolu des Turcs, on constate que, d'une façon ou d'une autre, ils sont discrètement exclus de la civilisation universelle à laquelle appartiennent les Grecs et les Romains de l'Antiquité. Le territoire turc est ainsi admiré, valorisé et approprié en tant que berceau de la civilisation, dont il conserve de nombreuses traces à travers ses vestiges gréco-romains et chrétiens, tandis que les habitants actuels de cet espace en sont expropriés en tant que nomades étrangers à la civilisation. Les Turcs sont pourtant bien présents sur ce territoire et les articles de presse ne sauraient en faire abstraction. Mais pour faire leur entrée, avec toutes leurs différences, dans le discours touristique, ils doivent passer par le crible de l'exotisation.

\section{À la découverte de l'Autre}

Le discours touristique construit l'Empire ottoman comme un Autre exotique. Suivant les traces des voyageurs européens du XIX ${ }^{\mathrm{e}}$ siècle, le journaliste part à la recherche de cet Empire exotique à travers la Turquie moderne et le reconstruit.

Qu'est-ce que l'exotisme ? Nous pouvons le définir comme une forme de relativisme, dans le sens où l'observateur empreint d'exotisme ne définit le pays ou la culture qu'il observe que par rapport à lui-même. Il décrit ainsi un pays dont «la seule caractéristique pertinente est qu'il ne soit pas le sien » (Todorov,1989 : 354). Selon Todorov, l'exotisme se fonde sur un jugement de valeur qui peut se résu- mer par : «Les autres sont mieux que nous ». Il s'oppose sur ce point au nationalisme qui affirme «nous sommes mieux que les autres », mais le rejoint en ce que, dans un cas comme dans l'autre, le regard porté sur l'autre renvoie invariablement à un «nous ». Dès lors, la définition de l'exotisme doit se faire de la manière suivante :

Il s'agit ici moins d'une valorisation de l'autre que d'une critique de soi, et moins de la description d'un réel que de la formulation d'un idéal. Personne n'est intrinsèquement autre ; il ne l'est que parce qu'il n'est pas moi ; en disant de lui qu'il est autre, je n'en ai encore rien dit vraiment; pis, je n'en sais rien et je n'en veux rien savoir, puisque toute caractérisation positive m'empêcherait de le maintenir dans cette rubrique purement relative, l'altérité (Todorov, 1989 : 355).

Pour que l'Autre reste enfermé dans son altérité, l'observateur « exotisant » doit donc veiller, dans ses descriptions, à privilégier certains contenus au détriment d'autres qui pourraient témoigner d'une trop grande proximité avec « nous ».

\section{L'exaltation de l'Empire ottoman}

S'agissant de la Turquie, le discours touristique produit par la presse française préserve l'altérité de l'autochtone « oriental » en limitant autant que possible son contenu au passé ottoman, réel ou imaginaire, de ce pays. Pour ce faire, les observateurs français s'inspirent aujourd'hui encore de l'exotisme conté et parfois vécu par les écrivains romantiques du $\mathrm{XIX}^{\mathrm{e}}$ siècle et du début du $\mathrm{XX}^{\mathrm{e}}$ siècle car, comme le fait remarquer Saïd, «L'Orient est une idée qui a une histoire et une tradition de pensée, une imagerie et un vocabulaire qui lui ont donné réalité et présence en Occident et pour l'Occident» (1997: 17). Ils délimitent le champ de leur discours à des objets tels que le sérail, le harem, les minarets, les mosquées, la voix du muezzin, les cafés et leurs fumeurs de narghilés, les vendeurs d'eau... Le simulacre de l'Empire ottoman survit ainsi à l'époque actuelle

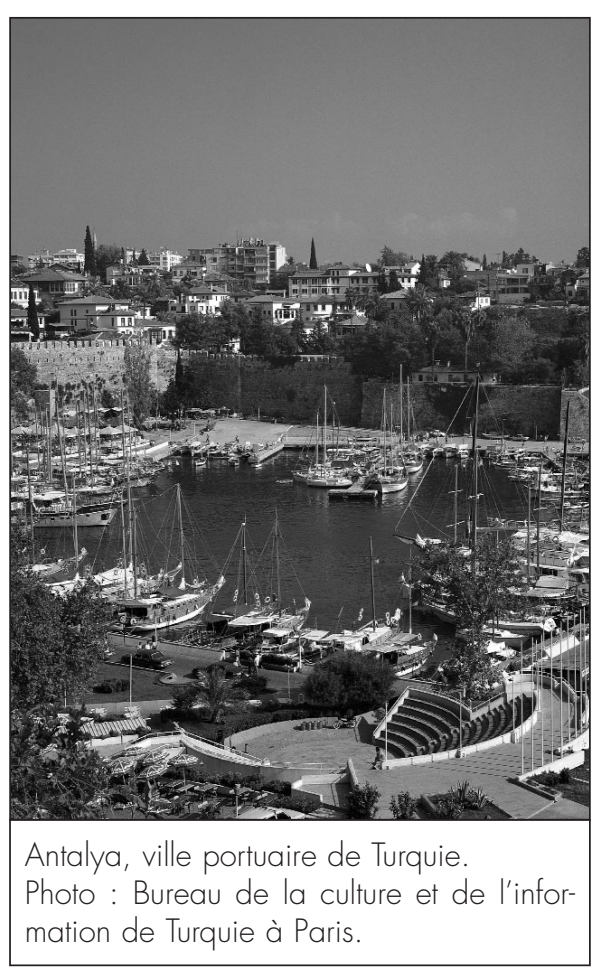

dans les représentations culturelles et politiques de la Turquie. Comme si, depuis la chute de ce dernier, rien n'avait bougé dans ce pays.

Les auteurs d'articles touristiques sur la Turquie procèdent à un inventaire détaillé de toutes les représentations exotiques nées à l'époque ottomane qui peuvent donner aujourd'hui un sens à la Turquie et fasciner l'imaginaire européen. Ils mentionnent ainsi le chant du muezzin, qui évoque pour eux les Mille et une nuits et transporte le visiteur bien loin de l'Europe : « [...] mais c'est un muezzin qui réveille la ville, la sort de son lit, un lamento de la Mecque, ses arabesques de la mille et unième nuit dans l'aube qui prend ses marques » (De Liederke : 1995). Dès que l'on quitte le registre exotique, les mosquées se transforment cependant en des lieux où l'islam fondamentaliste prend ses racines. On peut observer que les journalistes français constatent alors souvent le retour de l'Islam dans le pays de Mustafa Kemal, qui avait fondé la République turque sur le principe de la laiicité. Dès lors, la représentation de la Turquie actuelle met en avant l'identité musulmane de ce pays, sa différence culturelle avec l'Europe qui elle-même est 
désormais menacée par l'islamisme introduit en son sein par les immigrés turcs.

De retour à l'imagerie exotique, on trouve les traditionnels cafés turcs, dont la revue touristique Géo affirme qu'ils n'ont pas changé depuis un siècle :

Pierre Loti fut fasciné par le vieil Istanbul. Il y célébra le rite du narghileh, cette pipe à long tuyau ; aujourd'hui les clients des cafés turcs dans la même posture que les fumeurs du siècle précédent, gô̂tent à la douce torpeur où les plonge la fumée fortement aromatisée (Géo : 1989).

Autant que possible, l'Orient rêvé doit l'emporter sur le monde réel. La visite au palais des sultans ottomans n'a de sens que pour autant qu'elle permette à l'imagination du touriste européen de vagabonder dans leur intimité : «Un séjour à Istanbul, si court soit-il, comporte inévitablement une visite de Topkapi. Cette balade dans l'intimité des sultans [...] fait vagabonder l'imagination » (Prat, 1995). Au cœur de cet univers exotique siège le harem, thème incontournable du discours touristique qui en fait un indispensable témoin de la culture orientale :

Le sérail a hanté l'imagination de l'Europe qui a fantasmé sur ce jardin des délices peuplé de belles captives voluptueuses. Cet imaginaire aboutira au tableau le plus sensuel de la peinture française, le Bain turc d'Ingres, qui n'avait d'ailleurs jamais mis les pieds en Orient (Prat, 1995).

Bien qu'il appartienne à une époque révolue, le harem est sans cesse mobilisé par le discours touristique, où il continue d'évoquer la sensualité, la volupté, la richesse et le mystère qui ont nourri pendant des siècles l'imaginaire européen. Ce goût prononcé pour les descriptions romancées du harem ottoman trahit par ailleurs l'intimité des rapports entre exotisme et érotisme. La ville d'Istanbul dans son ensemble en vient ainsi parfois à être comparée à une femme sensuelle :

Voluptueusement couchée sur le divan de ses sept collines. Ainsi la décrit Théophile Gautier séduit par ce décor de Mille et une nuits [...] et que l'on imagine volontiers peuplé de courtisanes mystérieuses. Les fastes de l'Orient en trois syllabes : Istanbul (Tramard, 1992).

L'Orient est en effet décrit par le discours européen comme un lieu de rêve, vierge, séduisant et sensuel (Carelli, 1991 : 104) qui s'incarne, pour le regard masculin à l'origine du discours touristique, dans le corps d'une femme.

\section{Le désenchantement face à la modernité}

La Turquie contemporaine ne saurait pourtant, même dans le discours touristique, se réduire à de tels clichés exotiques. Au contraire du rédacteur publicitaire, le journaliste ne peut pas passer sous silence le fait que la modernité ait fait irruption au cœur de « l'Orient véritable », celui des écrivains romantiques du siècle dernier. Cette modernité, qu'elle s'exprime dans l'urbanisme, les modes de vie ou la massification du tourisme, est représentée de façon extrêmement dépréciative dans les articles de presse analysés. Elle se traduit notamment par la dégradation de l'environnement et du cadre de vie :

Aujourd'hui, l'intensité de la circulation dans le détroit, l'asphyxie des hauts fonds de la mer Noire par les rejets pétrochimiques russoukrainiens, les techniques modernes de la pêche [...], malmènent la faune [...] Et les cubes de béton avec vue-sur-bout-de-mer qui prolifèrent sur les hauteurs ne prodiguent que détresse architecturale (De Malefette, 1995).

On en vient à regretter les progrès du développement qui, en même temps qu'ils permettent à la population d'accéder à l'eau courante, défigurent le paysage urbain :

Percée de voies rapides, parsemée de nouvelles constructions, elle perd parfois le fil de son passé : les tours des nouveaux hôtels cinq étoiles sont là pour rappeler qu'il est bien révolu le temps où l'on souffrait ici de l'absence d'eau courante au cour d'un été déjà oriental (Prat, 1995).

L'adoption par les Turcs des modes de vie occidentaux condamne au désenchantement le visiteur européen qui, dans les ruelles d'Istanbul, part à la recherche de l'Orient :

Dans la rue de la Poule-qui-nepeut-pas-voler, les porteurs de Coca-Cola ont remplacé les porteurs d'eau [...] Quant aux fumeurs de narguilé (pipe à eau), ils disparaissent peu à peu tandis que les jeunes, plus de $55 \%$ de la population, fument plutôt la cigarette $d u$ cowboy, la Marlboro. Le soir à la télé, le petit peuple se gave de matchs de football (Lacontre, 1995).

$\mathrm{Si}$, comme le rappelle Guerlain, l'admiration qu'éprouve le touriste pour le pays qu'il visite est «la conséquence d'un regret ou d'une désolation à propos de son propre pays » (1996 : 59), la confrontation avec la réalité de la Turquie, où l'on trouve les mêmes comportements triviaux et la même américanisation des mœurs qu'en France, ne peut conduire qu'à une déception à la mesure de l'espoir de dépaysement nourri par le visiteur. L'arrivée du Coca-Cola et de la Marlboro ne signifie rien de moins que la mort de l'Orient, cette contrée imaginaire et exotique qui reste, pour le discours touristique, la Turquie véritable.

Ici s'esquisse en effet le refus de voir se perdre le fascinant «barbare » qu'était l'Empire ottoman. Selon Paul Dumont, spécialiste de la Turquie, les réformes successives des structures politiques entamées pendant l'Empire et poursuivies sous le régime républicain ont elles aussi été accueillies avec regret par certains Français :

Nostalgiques d'un passé "barbare », certaines sensibilités romantiques s'accommodaient mal de cette mise de la Turquie au goût $d u$ jour. Dans la foulée de Théophile Gautier et de Gérard de Nerval, voici par exemple ce qu'écrivait Pierre Loti peu de temps après l'a- 
doption de la constitution de 1876 : "Voilà cette pauvre Turquie qui proclame sa Constitution! Où allons-nous et dans quel siècle avons-nous reçu le jour? Un sultan constitutionnel, cela déroute toutes les idées qu'on m'avait inculquées sur l'espèce (1982:15).

Inspirés de ce discours romantique, les articles touristiques reviennent sans cesse à l'idée que la Turquie a perdu la mémoire de son passé oriental :

De plus en plus, la mémoire d'Istanbul et du Bosphore se fige dans les récits d'enfance d'habitants quinquagénaires. Ou les réminiscences d'une phrase tirée de la correspondance de Flaubert en 1850: "Au reste, il est temps de voir l'Orient car il s'en va, il se civilise (De Malefette,1995).

À l'exception de quelques vieux Stambouliotes de souche, élite souvent célébrée par les articles touristiques et élevée au rang de dépositaire de la mémoire de la ville sur le Bosphore, la société turque contemporaine est définie comme oublieuse de son passé et prompte à rejeter tout ce qui relève de la mémoire. On trouve ici l'idée d'un peuple nomade, destructeur des monuments hérités du passé, ceux des autres mais aussi les siens : "Si de tels avatars ont eu lieu, c'est parce que le goût pour le changement est une des caractéristiques du peuple turc. Ils ont toujours gardé l'âme nomade de leurs ancêtres. Il est dans leur mentalité de penser que tout ce qui est vieux est démodé » (Hernandez, 1994).

Cet oubli ou ce rejet du passé expose cependant les Turcs à un grave péril : celui de perdre leur identité. C'est ainsi que les journalistes français croient reconnaître dans le comportement des Turcs un certain mal-être lié à une crise d'identité, à la superposition de différentes identités mal assumées :

Comment être soi, ce prodige, au cour d'une Byzance d'islam, à l'ombre d'une église-mosquée, quand on est de surcroît un intel- lectuel coupé de ses racines d'Asie par le sabre d'Atatürk? Ô infortunés, tournés vers l'Occident sur le navire silencieux qui les mène vers l'Orient [...] Avec la modernité, sens et mémoire sombrent dans le Bosphore. Restent un joyau d'Orient serti dans un béton crasseux et une illisible calligraphie de clochers et de minarets (Franceschini,1995).

Une telle crise identitaire explique aussi bien, dans le discours journalistique, le retour en force d'un islam politique archaïque - tentative chimérique de retour à un passé imaginaire - que les lents progrès des démarches turques d'adhésion à l'Union européenne. De nombreux articles posent en effet la question : pourquoi, après tant d'années de convergence vers l'Europe, la Turquie n'est-elle toujours pas parvenue à se transformer en un pays européen ? La réponse tient dans le fait que ce pays, transformé en une petite Amérique proche-orientale, privé de sa texture originelle par l'occidentalisation, s'est perdu à mi-chemin de son passé et de son avenir. La Turquie est devenue un non-lieu, un espace innommable, "le milieu de nulle part, le rendez-vous de deux continents, le creuset et la projection de toutes sortes de rêves fous » (De Lidekerke,1995).

Milieu de nulle part, la Turquie est en définitive le lieu de tous les possibles. De ce télescopage jaillit un monde paradoxal, insaisissable, où le discours touristique trouve finalement son compte. De ces juxtapositions hasardeuses, les articles de la presse française tirent en effet un exotisme renouvelé, un «méta-exotisme » né du mariage improbable de l'Orient de Loti et de la modernité :

Encore faut-il croire aux fantômes, mais ceux d'Azyadé sont à chaque coin de rue d'Ortaköy ou de Péra. Presque aussi nombreux que les McDonalds, les cafés Pierre Loti. Le vrai existe vraiment; c'est une baraque en bois surplombant la Corne d'Or et les stèles endormies du cimetière d'Eyüp. Vue superbe - deux étoiles dans vos guides -, mais dans votre tasse un café turc infect parmi des sacs à dos scandinaves des groupes touristiques programmés comme un plan quinquennal [...] Pauvre Loti ! Désenchantement sur toute la ligne. Ici, mais sans doute est-ce cela aussi la magie d'Istanbul, son côté fin de règne, fin de siècle, khédive en exil, vieille star à qui on ne la fait pas. Ici, le vague à l'âme se déguise volontiers de kitsch et de babouches, d'un mauvais goût fastueux (De Lidekerke, 1995).

Dans le dédale des artères modernes et des venelles anciennes, le touriste est invité à suivre les traces de Nerval et de Lamartine, à chercher entre deux gratteciel les traces du simulacre de l'Orient passé, à se poser enfin la question : que reste-t-il de cet Orient fabuleux ? Il en reste tout et rien, un mélange insaisissable, mais charmant, affirme le discours touristique : «Pourtant, même bardé de toutes les cicatrices de sa modernité, nul autre décor ne sait mieux brocarder ses atouts. Et gratifier son visiteur, comme autrefois Flaubert, Nerval, Lamartine ou Loti, d'une rêverie orientaliste. Celle-ci reste intacte » (Guillaume,1994)

L'exotisme est mort, vive l'exotisme ! clament les articles à l'unisson. L'Orient, frappé de plein fouet par la modernité, ne ressemble certes plus aux descriptions qu'en avaient faites les voyageurs romantiques du siècle passé. La Turquie n'en reste pas moins exotique, à sa façon, faite de collages incongrus. Habilement recyclés, les descriptions des auteurs du XIX ${ }^{\mathrm{e}}$ siècle restent ainsi pour l'imaginaire français la grille de lecture indépassable de la Turquie contemporaine. Devant ce recyclage du discours exotique, il est possible de penser, avec Michel Foucault :

Tu n'as pas à craindre de commencer; nous sommes tous là pour te montrer que le discours est dans l'ordre des lois ; qu'on veille depuis son apparition ; qu'une place lui a été faite, qui l'honore mais le désarme; et que, s'il lui arrive d'avoir quelque pouvoir, c'est bien de nous, et de nous seulement, qu'il le tient (1971 : 9). 
Nilgun Tutal travaille à la faculté de communication de l'université d'Ankara en tant que chargée de cours. Ses recherches actuelles portent sur les théories de la communication et les questions d'altérité. Ces questions étaient également au centre de sa thèse de doctorat qui portait sur «les représentations de la Turquie dans la presse nationale et régionale française ».

\section{Notes}

1 Nous choisissons ici la France comme représentante de l'Europe et de la tradition orientaliste européenne. Selon Saïd, on peut en effet distinguer l'orientalisme ancien, européen, de l'orientalisme actuel, américain : «La position des Américains n'est pas tout à fait la même : pour eux, l'Orient a des chances d'être associé plutôt à l'Extrême-Orient (Chine et Japon pour l'essentiel). Pour leur part, les Français et les Anglais - et, dans une moindre mesure, les Allemands, les Russes, les Portugais, les Italiens et les Suisses - possèdent une longue tradition de ce que nous appellerions l'orientalisme, $[\ldots]$ qui exprime et représente cette partie (le Moyen-Orient et le ProcheOrient), culturellement et même idéologiquement, sous forme d'un mode de discours, avec, pour l'étayer, des institutions, un vocabulaire, un enseignement, une imagerie, des doctrines et même des bureaucraties coloniales et des styles coloniaux » (1997 : 14). Nous considérons ainsi qu'il est possible de rapprocher les représentations de l'Orient véhiculées par les orientalismes français, anglais ou d'une autre nation européenne et estimons hasardeux de tenter d'en attribuer la paternité à l'une ou l'autre de ces nations.

2 Cet article s'appuie sur les données d'une recherche réalisée dans le cadre d'une thèse de doctorat dont le corpus est constitué par des journaux et des magazines français nationaux et régionaux. Le Monde et ses suppléments de voyage, Le Figaro, Libération, Le Figaro Magazine, L'Express, Le Point, Géo, L'Alsace et Les Dernières Nouvelles d'Alsace (deux journaux régionaux) composent ce corpus. Pour limiter la taille de notre article, nous n'avons retenu qu'un nombre limité des 150 articles analysés dans le cadre de notre thèse, choisis selon leur représenta- tivité. Nous nous contentons ici de ne citer qu'un exemple d'article par thème répétitif recensé lors de notre recherche.

3 T.C Turizm Bakanligi, 2002.

4 Ibid.

5 Cf. L'Express, 23 mars 1995, et Le Figaro Magazine, 9 décembre 1995.

\section{Bibliographie}

« Istanbul au confluent de l'Europe et de l'Asie », Géo, n 184, juin 1994.

« La Turquie antique », Géo, n 97, 1987.

« La Turquie ottomane », Géo, n 23, 1989.

« La Turquie, plus on la connaît, plus on l'aime », Le Figaro Magazine, 4 juin 1994.

Admiral, J.R., et E.M. Lipiansky (1989), La communication interculturelle, Paris, Armand Colin.

Amossy, Ruth (1991), Les idées reçues, sémiologie du stéréotype, Paris, Édition Nathan.

Bécet, François (1995), «De la Turquie à l'Europe », L'Alsace, 12 mars.

Carelli, Mario (1991), « Évocation cavalière des images du Brésil en France : un héritage ambigu », dans Solange Parvaux et JeanRevel Mouroz (dir.), Images réciproques $d u$ Brésil et de la France, IHEAL.

Copeaux, Étienne (1997), Espace et Temps de la nation turque, Paris, CNRS Éditions.

Coutin, André (1987), «Une cité sans portes née sous le double signe du taureau et du vautour », Géo, n' 97, mars.

De Liedekerke, Arnould (1995), «Istanbul, La Sublime Porte », Le Figaro Magazine, 13 mai.

De Malefette, Arthur (1995), « Istanbul l'Européenne », L'Express, 4 mai.

Dumont, Paul (1982), « Le voyage en Turquie », Journal asiatique, $\mathrm{n}^{\circ}$ 3-4.

Fontana, Josep (1995), L'Europe en procès, Paris, Seuil.

Foucault, Michel (1971), L'Ordre du discours, Paris, Gallimard.

Franceschini, Jean-Paul (1995), « Le guetteur d'Istanbul », L'Express, 26 janvier.

Guerlain, Pierre (1996), Miroirs Transatlantiques, Paris, L'Harmattan.

Guillaume, Daniel (1994), « Sur le Bosphore, navigue la mémoire d'Istanbul », Géo, n 184, juin.

Guillebaud, Jean-Claude (1993), « Sur la route des croisades », Le Monde, 2 août.
Guillebaud, Jean-Claude (1994), « Une sublime porte entre l'Europe et l'Asie », Géo, $\mathrm{n}^{\circ} 184$, juin.

Héligon, Gérard (1994), « Istanbul ou la villemémoire », L'Alsace, 6 septembre.

Hentsch, Thierry (1988), L'Orient imaginaire, Paris, Édition de Minuit.

Hernandez, Florence (1994), « Pour les vieilles familles il a fallu changer d'époque », Géo, n 184.

Kilani, Mondher (1992), Introduction à l'anthropologie, Éditions Payot, Lausanne.

Lacontre, Robert (1995), « Pourquoi les Turcs rêvent-ils de revenir en Europe », Le Figaro Magazine, 9 décembre.

Lanfant, Marie-Françoise (1994), « Identité, mémoire, patrimoine et 'touristification' de nos sociétés », Sociétés, DUNOD, n 46.

Organisation Mondiale du Tourisme (2001), Aperçu mondial et actualité touristique, Madrid.

Prat, Véronique (1995), « Istanbul et l'art ottoman », Le Figaro Magazine, 13 mai.

Roux, Jean-Paul (1989), « Grandeur et Décadence de la dynastie d'Osman », Géo, $\mathrm{n}^{\circ}$ 123, mai.

Roux, Jean-Paul (1994), «Elle n'est plus capitale, mais elle reste la première », Géo $\mathrm{n}^{\circ} 184$, juin.

Saïd, Edward (1997), L'Orientalisme, l'Orient créé par l'Occident, Paris, Seuil.

Schneider, Marcel (1987), « Au soleil levant de l'Occident », Géo, n 97, mars.

Stoerkel, Jean-Marie (1995), « Le discours européen en Turquie », L'Alsace, 8 août.

T.C. Turizm Bakanligi, Arastirma ve Degerlendirme Dairesi Baskanligi, Sinir Giris Cikis Istatistikleri (2002), Haziran.

Todorov, Tzvetan (1989), Nous et les Autres, Paris, Seuil.

Tramard, Danielle (1992), « Le Bosphore pour miroir », Sans-Visa, supplément du Monde, 19 septembre.

Windisch, Uli (1989), « Représentations sociales, sociologie et sociolinguistique », dans Denise Jodelet, dir., Les représentations sociales, Paris, Presses universitaires de France. 